\title{
Lossless Compression of Ultraspectral Images Using Recursive Least Square with Constant Gain Vectors
}

\author{
Changguo Li ${ }^{*}$ \\ College of Fundamental Education, Sichuan Normal University, Chengdu 610068, \\ China \\ E-mail: 389224879@qq.com
}

\begin{abstract}
Based on the fact that the adaptation gain vector of the recursive least square (RLS) algorithm converges to zero as time increases when the parameters are time-invariant, a novel recursive least square with constant gain vectors (RLS-CGV) lossless compression method for ultraspectral images is proposed in this paper. Namely, an optimized recursive least square based on optimal number of prediction bands is introduced firstly. Then we randomly select a predetermined number of granules and use that set of granules for computing constant gain vectors. These gain vectors are used in the compression of all the other granules. Experimental results on publicly available NASA's Atmospheric Infrared Sounder (AIRS) data show that the proposed method outperforms the state-ofthe-art method, i.e., RLS, not only in terms of bit rate but also in terms of computing complexity.
\end{abstract}

Keywords: Recursive Least Square; Ultraspectral Images; Constant Gain Vector; Lossless Compression; AIRS

\section{Introduction}

The compression technique is an active research topic in remote sensing. It is generally known that ultraspectral instruments acquire images in thousands of narrow and continuous spectral bands. Moreover, the data volume of ultraspectral images has been drastically increased with the growing scientific and technological demands in spatial and spectral resolutions. For example, there are more than $25 \times 10^{6}$ pixels for each granule of AIRS (accounting for well over $40 \mathrm{MB}$ of data per single image). This poses a significant challenge to data transmission and storage. Therefore, there is an increasing need for highly performing image compression techniques. Typically, those compression techniques are classified into three modalities: lossless, lossy, and near-lossless. Since any degradation in images' quality is unacceptable, lossless compression has been one compression technique being widely used.

The predictive partitioned vector quantization (PPVQ) method [1] converts the ultraspectral sounder data into a Gaussian source using linear prediction. The residuals are partitioned based on bit depths. Vector quantization (VQ) is applied to each partition separately. In [2], independent component analysis (ICA) was applied for lossless compression of ultraspectral data. Wei and Huang [3] proposed a modified Tunstall code which grows an optimal non-exhaustive parse tree by assigning the complete codewords only to top probability nodes in the infinite tree, based on an infinitely extended parse tree. The linear prediction with optimal granule ordering (LP-OGO) method [4] computes linear prediction coefficients using a different granule. The optimal ordering problem of the granules is solved by using Edmonds' algorithm. In [5], a low-complexity linear prediction method (LP-CC) was proposed based on constant prediction coefficients. This method gives comparable results to the LP-OGO. Herrero and Ingle [6] presented a novel architecture that integrates both preprocessing and compression stages providing efficient 
lossy compression. A quantized-principal-component-analysis-based (ADQPCA) scheme [7] is presented by combining 3D prediction, positive mapping, and histogram packing using binary indexing vectors (positive packing) followed by a range coder. In [8], the use of linear prediction in the context of ultraspectral image compression from two particular points of view was explored; (1) it extends the traditional forward LP scheme and (2) it improves the precision of the mathematical modeling of the rate-distortion. The recursive least square (RLS) method [9] divides the compression process into two stages. First, the average value of four neighbor pixels of the current pixel is calculated as local mean, which is subtracted by the current pixel to eliminate correlation in the current band image. The residual produced by this step is called local difference. The local differences of the pixels which co-locate with the current pixel in previous bands form the input vector of the RLS filter, by which the prediction value of the current local difference is produced. Then, the prediction residual is sent to the adaptive arithmetic encoder.

In this paper, we propose a new recursive least square with constant gain vectors (RLSCGV) lossless compression method. First, we introduce an optimized RLS model based on optimal number of prediction bands. This model has a comparable average compression gain by spreading the spectral information from the current pixel to its neighbors until achieving a global stable state on the whole image. Then, according to the characteristic of the adaptation gain vector converging to zero, we randomly select a predetermined number of granules and use that set of granules for computing constant gain vectors. After that, we use RLS-CGV to compress all the granules. The rest of this paper is organized as follows. Section II describes the recursive least square with constant gain vectors (RLS-CGV) compression method. Experimental results are given in Section III. Section IV concludes this paper.

\section{RLS-CGV}

\section{A. Recursive Least Square (RLS)}

As an adaptive filtering algorithm, RLS can automatically adjust the current filter parameters using the previously obtained ones, in order to adapt the unknown or timevarying statistical properties of the signal and noise, and achieve optimal filtering. For the above reasons, the applications of RLS have drawn wide attention in recent years. In [9], RLS has been proved to be an extremely powerful compression tool for remote sensing images, which has strong correlations on both spectral and spatial dimensions, and leads to the state-of-the-art performance. To define the problem in mathematical terms for compression stage, let $s_{z}(t)$ represent the current pixel, where $\mathrm{x}$ and $\mathrm{y}$ are the coordinates of the current pixel in the current band, $\mathrm{W}$ and $\mathrm{H}$ are the image's width and height, and $t=W_{y}+x$. For the first band, the intraband estimate of pixel $s_{z}(t)\left(\tilde{s}_{z}(t)\right)$ is given as follows:

$$
\begin{gathered}
\tilde{s}_{z}(t)=\left(s_{z}^{W}(t)+s_{z}^{N W}(t)+s_{z}^{N}(t)+s_{z}^{N E}(t)\right) / 4 \\
d_{z}(t)=s_{z}(t)-\widetilde{s}_{z}(t)
\end{gathered}
$$

where $s_{z}^{I I}(t), s_{z}^{N I}(t), s_{z}^{N}(t)$, and $s_{z}^{N E}(t)$ denote four neighboring pixels, respectively. For the other bands, the RLS filter is adopted to conduct the interband prediction by the following model:

$$
e_{z}(t)=d_{z}(t)-\left\lfloor d_{z}(t) \boldsymbol{W}^{T}(t-1)\right\rfloor
$$

where the input vector $d_{z}(t)=\left[d_{z-1}(t), d_{z-2}(t), \cdots, d_{z-p}(t)\right]$, the weight vector $w(t)=\left[W_{1}(t), \cdots W_{p}(t)\right], \mathrm{p}$ is the number of prediction bands used for predicting the current band, $\mathrm{w}(0)=[0]$, and $\mathrm{t}=1$. The gain vector $\mathrm{k}(\mathrm{t})$, the inverse correlation matrix $\mathrm{P}(\mathrm{t})$, and the weight vector $\mathrm{w}(\mathrm{t})$ are updated as follows:

$$
k^{T}(t)=\frac{P(t-1) \boldsymbol{d}_{z}^{T}(t)}{1+\boldsymbol{d}_{z}(t) P(t-1) \boldsymbol{d}_{z}^{T}(t)}
$$




$$
\begin{gathered}
P(t)=P(t-1)-k^{T}(t) \boldsymbol{d}_{z}(t) P(t-1) \\
\boldsymbol{W}(t)=\boldsymbol{W}(t-1)+k(t) e_{z}(t)
\end{gathered}
$$

where $P(0)=\delta I_{P}, \delta=0.0001, I_{p}$ is the p-order identity matrix. Then $\mathrm{t}=\mathrm{t}+1$, and the next pixel is executed the same interband prediction. When $t$ is larger than $\mathrm{W} \times \mathrm{H}$, the procedure of current band prediction is ended.

B. Optimized RLS Based on Optimal Number of Prediction Bands

In ultraspectral images, spectral correlations are usually stronger than spatial correlations. It is expected that compression methods based on spectral correlation can work well. Previous works indicate that by taking advantage of the spectral correlation, compression performance can be significantly improved [6]-[8], and on the basis, prediction bands are further explored to optimize the compression results [4]-[5].

Since the spatial location of the current pixel is known, this aspect can be included in the RLS model to retain the spectral information of the current pixel and spread them to neighboring pixels. Thus, the compression results in bits-per-pixel (BPP) of the RLS model can be further reduced by spreading the spectral information from the current pixel to its neighbors until achieving a global stable state on the whole image. In order to obtain the optimal number of prediction bands for RLS, we varied the number of prediction bands from 50 to 600 using a stride value of 50 in the first test. For test data, we used NASA's AIRS images (details of the dataset will be given in Section III). The results from tests are quantitatively shown in Table 1 . The number of prediction bands is shown in column 1. Columns 2-11 show results using ten granules. For this test purpose, we are only interested in finding the optimal value for the number of prediction bands $\mathrm{p}$. As can be seen form Table 1, the average compression bits-per-pixel reaches its minimum at 300 previous prediction bands. Thus, we take the 300 previous bands of each granule for computing the prediction.

\section{Table 1. Compression Results of Different Previous Prediction Bands (Bits per Pixel)}

\begin{tabular}{|c|l|l|l|l|l|l|l|l|l|l|c|}
\hline $\begin{array}{c}\text { number of } \\
\text { bands }\end{array}$ & 9 & 16 & 60 & 82 & 120 & 126 & 129 & 151 & 182 & 193 & Average \\
\hline 50 & 4.35 & 4.37 & 4.36 & 4.40 & 4.29 & 4.34 & 4.28 & 4.37 & 4.41 & 4.35 & 4.35 \\
\hline 100 & 4.34 & 4.38 & 4.35 & 4.39 & 4.27 & 4.34 & 4.26 & 4.36 & 4.37 & 4.32 & 4.34 \\
\hline 150 & 4.34 & 4.38 & 4.35 & 4.39 & 4.38 & 4.34 & 4.26 & 4.38 & 4.38 & 4.32 & 4.35 \\
\hline 200 & 4.34 & 4.38 & 4.35 & 4.51 & 4.27 & 4.35 & 4.26 & 4.38 & 4.38 & 4.32 & 4.35 \\
\hline 250 & 4.33 & 4.37 & 4.35 & 4.38 & 4.26 & 4.33 & 4.25 & 4.39 & 4.37 & 4.31 & 4.33 \\
\hline 300 & 4.32 & 4.36 & 4.34 & 4.37 & 4.25 & 4.32 & 4.24 & 4.38 & 4.36 & 4.30 & 4.32 \\
\hline 350 & 4.32 & 4.36 & 4.34 & 4.38 & 4.25 & 4.32 & 4.24 & 4.38 & 4.36 & 4.30 & 4.32 \\
\hline 400 & 4.32 & 4.36 & 4.34 & 4.38 & 4.25 & 4.32 & 4.24 & 4.38 & 4.36 & 4.30 & 4.32 \\
\hline 450 & 4.36 & 4.40 & 4.38 & 4.42 & 4.28 & 4.36 & 4.24 & 4.39 & 4.37 & 4.31 & 4.35 \\
\hline 500 & 4.36 & 4.40 & 4.38 & 4.42 & 4.27 & 4.36 & 4.24 & 4.38 & 4.37 & 4.31 & 4.34 \\
\hline 550 & 4.36 & 4.40 & 4.39 & 4.42 & 4.27 & 4.36 & 4.25 & 4.37 & 4.37 & 4.32 & 4.35 \\
\hline 600 & 4.36 & 4.40 & 4.39 & 4.42 & 4.27 & 4.35 & 4.24 & 4.38 & 4.37 & 4.31 & 4.35 \\
\hline
\end{tabular}

\section{Recursive Least Square with Constant Gain Vectors}

As is well known, a large number of physical processes in the real world are not timeinvariant, such as ultraspectral image process. Thus the adaptation gain vector converges to zero as time increases [10]. Meanwhile, we know that the adaptation gain vector is only the intermediate variable used to meet the computing needs in the RLS filter. Its computation involves three multiplication, one addition, and one division operations, which has been the most time-consuming part. For these reasons, we use optimized RLS based on optimal number of prediction bands as a case study to further optimize its computing complexity by adopting the constant gain vectors. 
In order to demonstrate the trend of the original adaptation gain vectors, we compress the AIRS-Granule-9 image using the 300 previous prediction bands, and reserve its adaptation gain vectors. Figure 1 illustrates the trend of each mean component (for example, the 100th mean component) for each band. From Figure 1, it can be found that the adaptation gain vector converges to zero as time increases, and each band has the same trend. Thus, we will carry out the ultraspectral compression using RLS with constant gain vectors, instead of adaptation gain vectors.

We now continue to pay attention to the bit rate. The optimized RLS based on optimal number of prediction bands, it is true, can effectively reduce the compression results in terms of BPP on AIRS images. Nevertheless, the number of the base granules and their component granules used to calculate the constant gain vectors still have an important influence on the compression results. Two small examples of the different number of base granules and the different combination of base granules for computing the constant gain vectors are presented next, respectively.

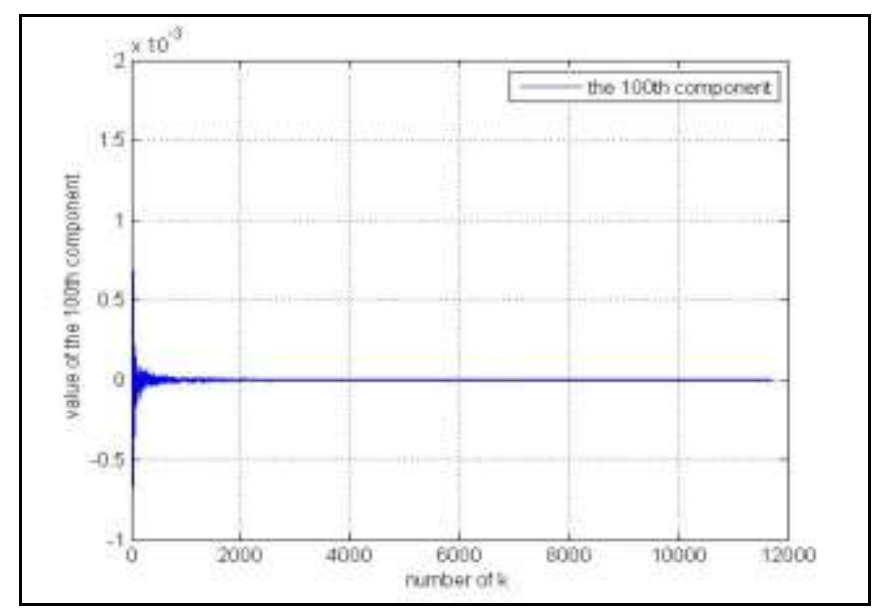

Figure 1. The Trend of the 100th Mean Component for each Band of AIRSGranule-9 Image

For both examples, the compression results are shown in Tables 2 and 3 respectively. As can be seen from Table 2, three base granules are sufficient to utilize the statistical properties of a set of AIRS granules for the compression of another set of granules. In Table 3, the final row shows the average values, which are fairly stable with regard to the base granules set. This indicates that we can expect the method to perform well on novel data. 


\section{Table 2. Compression Results of RLS Using Different Number of Base} Granules (Bits per Pixel)

\begin{tabular}{|c|c|c|c|c|c|c|c|c|c|c|c|}
\hline $\begin{array}{c}\text { Number of } \\
\text { base granules }\end{array}$ & 9 & 16 & 60 & 82 & 120 & 126 & 129 & 151 & 182 & 193 & Average \\
\hline 2 & 4.42 & 4.46 & 4.45 & 4.48 & 4.33 & 4.41 & 4.30 & 4.44 & 4.43 & 4.37 & 4.41 \\
\hline 3 & 4.37 & 4.42 & 4.40 & 4.43 & 4.27 & 4.35 & 4.25 & 4.39 & 4.39 & 4.32 & 4.36 \\
\hline 5 & 4.40 & 4.42 & 4.41 & 4.46 & 4.30 & 4.39 & 4.27 & 4.40 & 4.40 & 4.34 & 4.38 \\
\hline 6 & 4.42 & 4.42 & 4.43 & 4.46 & 4.31 & 4.40 & 4.29 & 4.40 & 4.40 & 4.36 & 4.39 \\
\hline 7 & 4.42 & 4.45 & 4.45 & 4.49 & 4.33 & 4.41 & 4.31 & 4.43 & 4.44 & 4.36 & 4.41 \\
\hline 8 & 4.42 & 4.46 & 4.46 & 4.47 & 4.34 & 4.41 & 4.30 & 4.43 & 4.43 & 4.37 & 4.41 \\
\hline 9 & 4.43 & 4.48 & 4.45 & 4.49 & 4.33 & 4.43 & 4.31 & 4.45 & 4.44 & 4.38 & 4.42 \\
\hline
\end{tabular}

Table 3. Compression Results of RLS Using Different Combination of Base Granules (Bits per Pixel)

\begin{tabular}{|c|c|c|c|c|c|c|c|c|c|c|}
\hline 9 & 16 & 60 & 82 & 120 & 126 & 129 & 151 & 182 & 193 & Average \\
\hline 4.35 & 4.40 & 4.38 & 4.41 & 4.25 & 4.33 & 4.23 & 4.37 & 4.37 & 4.30 & 4.34 \\
\hline 4.37 & 4.41 & 4.41 & 4.42 & 4.27 & 4.36 & 4.24 & 4.39 & 4.40 & 4.32 & 4.36 \\
\hline 4.36 & 4.42 & 4.38 & 4.42 & 4.26 & 4.33 & 4.26 & 4.38 & 4.39 & 4.30 & 4.35 \\
\hline 4.35 & 4.39 & 4.37 & 4.41 & 4.26 & 4.33 & 4.24 & 4.37 & 4.38 & 4.29 & 4.34 \\
\hline 4.38 & 4.40 & 4.40 & 4.43 & 4.28 & 4.35 & 4.24 & 4.38 & 4.41 & 4.32 & 4.36 \\
\hline 4.35 & 4.43 & 4.39 & 4.41 & 4.27 & 4.33 & 4.26 & 4.37 & 4.38 & 4.31 & 4.35 \\
\hline 4.37 & 4.42 & 4.40 & 4.42 & 4.31 & 4.36 & 4.24 & 4.37 & 4.38 & 4.32 & 4.36 \\
\hline 4.36 & 4.41 & 4.42 & 4.41 & 4.28 & 4.36 & 4.25 & 4.39 & 4.40 & 4.31 & 4.36 \\
\hline 4.37 & 4.42 & 4.37 & 4.43 & 4.26 & 4.32 & 4.26 & 4.39 & 4.38 & 4.30 & 4.35 \\
\hline 4.36 & 4.39 & 4.39 & 4.42 & 4.24 & 4.33 & 4.23 & 4.36 & 4.37 & 4.30 & 4.34 \\
\hline \hline 4.36 & 4.41 & 4.39 & 4.42 & 4.27 & 4.34 & 4.25 & 4.38 & 4.38 & 4.30 & 4.35 \\
\hline
\end{tabular}

The prediction phase is followed by an adaptive arithmetic coder (AAC). Namely, after prediction, the difference between the predicted and original values is computed. The difference is further entropy-coded using the AAC.

\section{Experimental Results}

In order to test the performance of our proposed method, some experiments are carried out. The ultraspectral images for test are the NASA's AIRS radiance data. The AIRS data cover 2378 infrared channels in the 3.74-15.4 um region of the spectrum. The data gathered during the $24 \mathrm{~h}$ period are divided into 240 granules. Each granule consists of 135 scan lines and 90 cross-track footprints per scan line. Ten selected test data granules are available via anonymous ftp (ftp://ftp.ssec.wisc.edu/pub/bormin/Count). In order to make the test more generic to other ultraspectral sounders, 270 bad channels have been excluded from the publicly available test data. The bit depth of a channel rages from 12 to 14 bits for different channels. Figure 2 shows five representative bands of AIRS granule 9. All experiments were run on a $4.0 \mathrm{GHz}$ Intel Core i7-4790K, and were performed using the 64-bit Microsoft Windows 7 operating system. 


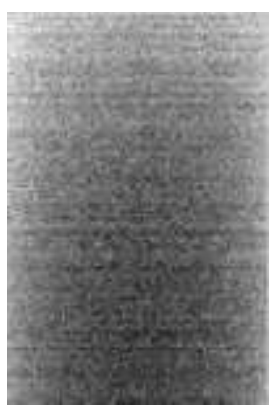

(a) Band 1

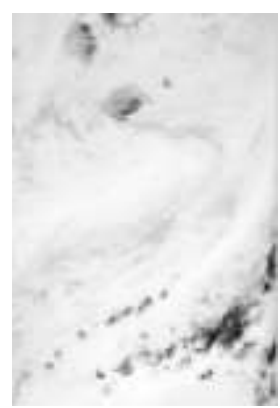

(b) Band 500

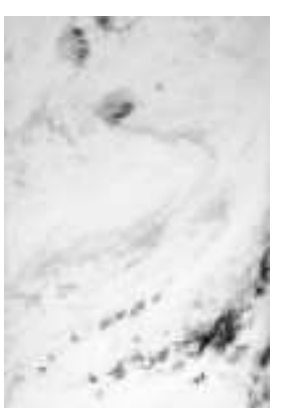

(c) Band 1000

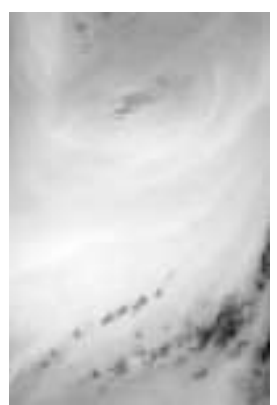

(d) Band 1500

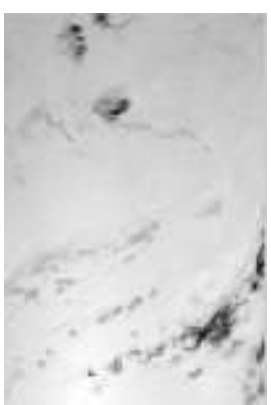

(e) Band 2000

Figure 2. AIRS Granule 9

The first experiment was carried out to test the compression results in BPP of the proposed method compared to several other methods. The compression results are quantitatively shown in Table 4. For the RLS-CGV method, we report the average results from Table 3. Thus, we get a more accurate picture of how the method will perform on novel data. The results indicate that our proposed method achieves the best compression performance in terms of bit rate, and outperforms the state-of-the-art RLS method by more than 0.11 BPP. Exploring its reason, it can be found that this is primarily attributable to the use of optimal prediction bands. Its essence is that RLS-CGV spreads the spectral information from the current pixel to its neighbors until achieving a global stable state on the whole image.

Table 4. Compression Results of Several Other Compression Methods (Bits per Pixel)

\begin{tabular}{|c|c|c|c|c|c|c|c|c|c|c|c|}
\hline $\begin{array}{c}\text { granule } \\
\text { algorithm- }\end{array}$ & 9 & 16 & 60 & 82 & 120 & 126 & 129 & 151 & 182 & 193 & Average \\
\hline LP & 5.17 & 5.09 & 5.312 & 4.93 & 5.16 & 5.32 & 4.92 & 5.24 & 5.36 & 5.29 & 5.18 \\
\hline JPEG-LS & 5.24 & 5.14 & 5.37 & 5.00 & 5.20 & 5.37 & 5.00 & 5.28 & 5.44 & 5.35 & 5.24 \\
\hline LUT & 5.63 & 5.39 & 5.73 & 5.30 & 5.65 & 5.68 & 6.11 & 5.81 & 5.94 & 5.81 & 5.71 \\
\hline FL & 4.58 & 4.64 & 4.60 & 4.65 & 4.11 & 4.57 & 4.50 & 4.62 & 4.60 & 4.52 & 4.58 \\
\hline IP3 & 4.52 & 4.57 & 4.53 & 4.57 & 4.42 & 4.48 & 4.41 & 4.53 & 4.52 & 4.45 & 4.50 \\
\hline RLS & 4.45 & 4.51 & 4.52 & 4.53 & 4.39 & 4.45 & 4.36 & 4.48 & 4.48 & 4.42 & 4.46 \\
\hline RLS-CGV & 4.36 & 4.41 & 4.39 & 4.42 & 4.27 & 4.34 & 4.25 & 4.38 & 4.38 & 4.30 & 4.35 \\
\hline
\end{tabular}

In order to evaluate the computing complexity of our method, we run all methods on the considered computer platform, in which one out of four processor cores is used. The average compression times of JPEG-LS and IP3 are $3.09 \mathrm{~s}$ and $42.67 \mathrm{~s}$, respectively. They represent the lowest and the highest computing complexities. For RLS, its most timeconsuming part is the computation of the adaptation gain vector. Meanwhile, it requires updating formulas (1) to (6) when compressing each pixel. Thus tentatively compressing a granule would take $29.43 \mathrm{~s}$. Using 240 granules, the computation would take 1.96 hours. In comparison, RLS-CGV would require 1.62 hours for the compression of 240 granules. That means that RLS-CGV gets a $2.47 \%$ decrease in BPP while the computation times are reduced by $17.35 \%$. Therefore, it is worth mentioning that RLS-CGV is a more practical compression method than the state-of-the-art method RLS.

\section{Conclusion}

In this paper, a novel efficient method (RLS-CGV) of lossless compression for ultraspectral images is proposed using recursive least square with constant gain vectors. The experimental results on publicly available NASA's AIRS data show that RLS-CGV gets $2.47 \%$ and $17.35 \%$ decreases in terms of bit rate and computation time, respectively, 
compared to the state-of-the-art method RLS. Because the scheme based on optimal number of prediction bands features better compression results at the expense of higher computation times, which have caused the optimization of the computation time being not significant, future work will focus on further reduction of the computation time by algorithm optimization.

\section{Acknowledgments}

This work is supported by the project of the Science and Technology Department in Sichuan province (2017JY0326), the Research Foundation of the Sichuan Department of Education (15ZB0044), and the Research Foundation of Sichuan Normal University (2015KYQD312).

\section{References}

[1] B. Huang, A. Ahuja, and H. Huang, "Lossless compression of ultraspectral sounder data", in Hyperspectral Data Compression, G. Motta, F. Rizzo, and J. Storer, Eds. Berlin, Germany: SpringerVerlag, (2006), pp. 75-106.

[2] S. Wei and B. Huang, "Application of independent component analysis to lossless compression of 3D ultraspectral sounder data", in Proc. Asia Pacific Conf. Commun., (2007), pp. 197-199.

[3] S. Wei and B. Huang, "Ultraspectral sounder data compression using the non-exhaustive Tunstall coding," in Satellite Data Compression, Communication, and Processing IV, San Diego, California, USA, 7084: 708406-708406-9, (2008).

[4] J. Mielikainen and P. Toivanen, "Optimal granule ordering for lossless compression of ultraspectral sounder data," Proc. SPIE, vol. 7084, (2008), pp. 708404.

[5] J. Mielikainen and P. Toivanen, "Lossless compression of ultraspectral sounder data using linear prediction with constant coefficients," IEEE Geoscience and Remote Sensing Letters, vol. 6, no. 3, (2009), pp. 495-499.

[6] R. Herrero and V. K. Ingle, "lossy compression of ultraspectral images: integrating preprocessing and compression stages", Signal, Image and Video Processing, vol. 8, no. 8, (2014), pp. 1569-1580.

[7] H. Chen, B. Huang, and Y. Zhang, "Adaptive quantized PCA with 3D prediction and positive packing for lossless compression of ultraspectral sounder data," International Journal of Remote Sensing, vol. 36, no. 6, (2015), pp. 1689-1704.

[8] R. Herrero and V. K. Ingle, "Backward and forward linear prediction applied to ultraspectral image processing”, Signal, Image and Video Processing, vol. 10, no. 4, (2016), pp. 639-646.

[9] J. W. Song, Z. W. Zhang, X. M. Chen, "Lossless compression of hyperspectral imagery via RLS filter," Electronics Letters, vol. 49, no. 16, (2013), pp. 992-994.

[10] D. J. Park, and B. E. Jun, "Selefperturbing recursive least squares algorithm with fast tracking capability," Electronics Letters, vol. 28, no. 6, (1992), pp. 558-559. 
International Journal of Signal Processing, Image Processing and Pattern Recognition Vol. 10, No. 7 (2017) 